\title{
Max-min Fairness for Multicast Multigroup Multicell Transmission under Backhaul Constraints
}

\author{
Quang-Doanh Vu, Kien-Giang Nguyen, and Markku Juntti \\ Centre for Wireless Communications, University of Oulu, P.O.Box 4500, FI-90014 University of Oulu, Finland \\ Email: \{quvu,nguyen.k.giang,markku.juntti\}@ee.oulu.fi
}

\begin{abstract}
We consider multicell downlink transmission where multi-antenna base stations (BSs) collaborate in transferring data to multiple cochannel multicast groups. Our aim is to design a joint transmitter selection and cooperative precoding scheme which maximizes the minimum received data rate among all receivers under constraints on the individual BS transmit power budgets as well as backhaul link capacity. The problem is naturally cast as a mixed Boolean nonconvex program whose global optimal solution is difficult to achieve. To solve this problem locally, we first use difference-convex (DC) functions to represent the Boolean variables, and further transform the problem into an equivalent but more tractable formulation. We then propose an iterative algorithm which provably converges to stationary solutions. Numerical results are provided to demonstrate the superior performance of the proposed method.
\end{abstract}

\section{INTRODUCTION}

The evolution of wireless communications with the continuous increase in capacity allows operators to provide various kinds of services to subscribers. The development of user devices (e.g. smartphones) with high-quality media capabilities boosts the growth of wireless service demand in general, and multicast transmission in particular. Therein common information (e.g, news, video clips, TV shows) is simultaneously delivered to a group of users in a defined area [1], [2], and those have been become a feature in current wireless communication standards, e.g. the Long-Term Evolution (LTE) [3]. Multicast transmissions has also received significant research attention in recent years, e.g. in multicell networks [4] or cognitive communications [5].

Coordinated multi-point (CoMP) transmission is one of the key technologies to improve the capacity of wireless networks, particularly for the cell edge users. In this context, transmitters collaborate in designing a precoder operating as a large virtual multiple-input multiple-output (MIMO) system (i.e. joint beamforming) [6]. However, this technique requires that user data is available at all transmitters, and thus, increasing the amount of information transmitted over the backhaul links connecting the radio controllers and base stations (BSs). Unfortunately, capacity of the backhaul link is limited regardless the physical medium (e.g copper, optical fiber or microwave radio wireless) is deployed [7]. A solution for the challenge is properly selecting the sets of serving transmitters such that the number of duplicate versions of data transferred over the backhaul link is reduced. This approach has been considered in many current works with different objectives, e.g. sum rate maximization [8], or joint backhaul and transmit power minimization [9], [10].

In this paper, we consider multicell wireless networks where multi-antenna BSs cooperate on transmitting data to some multicast groups. Different from [10], where the transmit power and backhaul cost are jointly minimized, we aim at maximizing the minimum data rate among multicast groups subject to the backhaul capacity by developing a joint transmitter selection and precoder design. Due to the NP-hard complexity of the multicast fairness problem and the combination of transmitter selection, the design problem is cast as a mixed Boolean nonconvex program which is also intractable. Hence, to find the global optimal solution is a difficult challenge and not practical interest. As such, we develop a low-complexity method which solves the problem locally. For this purpose, we first tackle the discrete parts by using difference-convex (DC) functions to convert the Boolean variables into continuous ones. Then, the problem is equivalently transformed such that the convexity is more exposed. Subsequently, we propose an iterative procedure where a second-order cone program (SOCP) is solved in each iteration. In addition, we prove that the proposed method converges to stationary points of the equivalent problem. Numerical results are carried out to empirically evaluate the effectiveness of the proposed method.

The rest of the paper is organized as follows. System model and problem formulation are described in Section II. Section III presents the problem transformation and the proposed iterative algorithm. Numerical results and discussions are provided in Section IV. Finally, Section V concludes the work.

Notation: Standard notations are used in this paper. Bold lower and upper case letters represent vectors and matrices, respectively; $\|\cdot\|_{2}$ represents the $l_{2}$ norm; $|\cdot|$ represents the absolute value; $\mathbb{C}^{a \times b}$ represents the space of complex matrices of dimensions given in superscript; $\mathcal{C N}(0, c)$ denotes a complex Gaussian random variable with zero mean and variance $c$; $\Re(\cdot)$ represents real part of the argument. Other notations are defined at their first appearance.

\section{System Model And Problem Formulation}

Consider a wireless communication system where $B$ BS transmitters jointly serve $G$ multicast groups consisting $U$ single-antenna receivers (users) and each belongs to only one group [2]. An example of the considered system model is displayed in Fig. 1. Let $g_{i}$ indicate receiver $i$ of group $g$ and $L_{b}, L_{b} \geq 2$, denote the number of antennas implemented at 


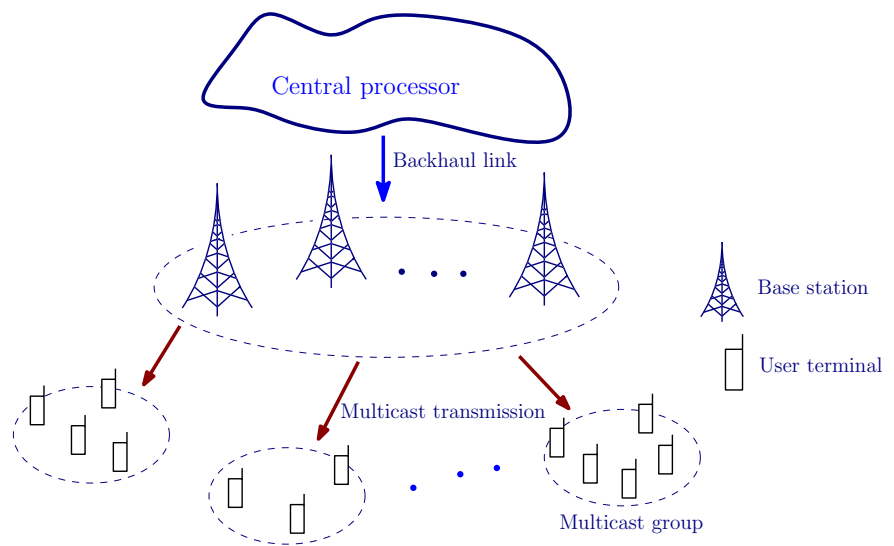

Figure 1. An example of multicast multigroup cooperative transmissions with backhaul link connecting central processor with BSs.

transmitter $b$. Let $\mathbf{h}_{g_{i} b} \in \mathbb{C}^{1 \times L_{b}}$ be the channel (row) vector from transmitter $b$ to receiver $g_{i}$, and $\mathbf{w}_{g b} \in \mathbb{C}^{L_{b} \times 1}$ be the precoder for group $g$ at transmitter $b$. With these introduced notations and under flat fading channels, the received signal at receiver $g_{i}$ is written as

$$
y_{g_{i}}=\sum_{b=1}^{B} \mathbf{h}_{g_{i} b} \mathbf{w}_{g b} x_{g}+\sum_{b=1}^{B} \sum_{\substack{k=1, k \neq g}}^{G} \mathbf{h}_{g_{i} b} \mathbf{w}_{k b} x_{k}+z_{g_{i}}
$$

where $z_{g_{i}} \sim \mathcal{C N}\left(0, \sigma_{g_{i}}^{2}\right)$ is the additive white Gaussian noise (AWGN) at receiver $g_{i}$ and $x_{g}$ is the normalized complex data symbol intended for group $g$. For ease of description, let $\mathbf{w}_{g} \triangleq\left[\mathbf{w}_{g 1}^{T}, \mathbf{w}_{g 2}^{T}, \ldots, \mathbf{w}_{g B}^{T}\right]^{T} \in \mathbb{C}^{\left(\left(\sum_{b=1}^{B} L_{b}\right) \times 1\right)}$ and $\mathbf{h}_{g_{i}} \triangleq\left[\mathbf{h}_{g_{i} 1}, \mathbf{h}_{g_{i} 2}, \ldots, \mathbf{h}_{g_{i} B}\right] \in \mathbb{C}^{\left(1 \times\left(\sum_{b=1}^{B} L_{b}\right)\right)}$ which are the beamformer vector and channel vector from all transmitters to receiver $g_{i}$, respectively. Then, based on (1), the signalto-interference-plus-noise ratio (SINR) at receiver $g_{i}$ is given by

$$
\gamma_{g_{i}}=\frac{\left|\mathbf{h}_{g_{i}} \mathbf{w}_{g}\right|^{2}}{\sum_{k=1, k \neq g}^{G}\left|\mathbf{h}_{g_{i}} \mathbf{w}_{k}\right|^{2}+\sigma_{g_{i}}^{2}} .
$$

In the context of multicast transmission, the common information rate is determined by the weakest link. Thus the common data rate transmitted to receivers in group $g$ is given by [1]

$$
R_{g}=\log \left(1+\min _{\forall i}\left\{\gamma_{g_{i}}\right\}\right) \text {. }
$$

We suppose that multicast data is transported from the central processor to the transmitters via the backhaul link [8], [9], [11]. Thus, the corresponding total backhaul rate requirement is [10]

$$
f_{\mathrm{BH}} \triangleq \sum_{g=1}^{G}\left|\mathcal{B}_{g}\right| R_{g}
$$

where $\mathcal{B}_{g} \in\{1,2, \ldots, B\}$ is the set of transmitters serving group $g$, and $|\mathcal{B}|$ denotes the cardinality of set $\mathcal{B}$. Assuming that the capacity limitation of the backhaul link is given by parameter $\bar{C}$, then we have $f_{\mathrm{BH}} \leq \bar{C}$. By (4), we can see that the total backhaul rate requirement $f_{\mathrm{BH}}$ depends on data rate $R_{g}$ as well as sets of serving BSs $\mathcal{B}_{g}, \forall g$. That is to say, besides designing the precoding vector, properly choosing the serving BSs for each group can also improve the common information rate. From this perspective, let us introduce the selection variables $d_{g b} \in\{0,1\}$ where $d_{g b}=1$ indicates that group $g$ is served by BS $b$ and $d_{g b}=0$ otherwise. Then, the problem of max-min fairness data rate for multicast multigroup systems can be formulated as

$$
\begin{gathered}
\underset{\mathbf{w}_{g}, p_{g b}, d_{g b}}{\operatorname{maximize}} \min _{\forall g_{i}}\left\{\frac{\left|\mathbf{h}_{g_{i}} \mathbf{w}_{g}\right|^{2}}{\sum_{k=1, k \neq g}^{G}\left|\mathbf{h}_{g_{i}} \mathbf{w}_{k}\right|^{2}+\sigma_{g_{i}}^{2}}\right\} \\
\text { subject to } \sum_{g=1}^{G}\left(\sum_{b=1}^{B} d_{g b}\right) R_{g} \leq \bar{C} \\
\left\|\mathbf{T}_{b} \mathbf{w}_{g}\right\|_{2}^{2} \leq d_{g b} p_{g b}, \forall g, b \\
\sum_{g=1}^{G} p_{g b} \leq \bar{P}_{b}, \forall b \\
d_{g b} \in\{0,1\}, \forall g, b
\end{gathered}
$$

where $\mathbf{T}_{b} \triangleq\left[\mathbf{0}_{L_{b} \times \sum_{t=1}^{b-1} L_{t}}, \mathbf{I}_{L_{b}}, \mathbf{0}_{L_{b} \times \sum_{t=b+1}^{B} L_{t}}\right]$ and $p_{g b} \geq 0$ is the soft power variable, which is introduced to force precoder $\mathbf{w}_{g b}$ to be zero when transmitter $b$ does not serve group $g$ (i.e $(5 \mathrm{c})$ ). We also consider the power constraint at each transmitter in $(5 d)$. The backhaul constraint in $(5 b)$ is written based on (4) since $\left|\mathcal{B}_{g}\right|=\sum_{b=1}^{B} d_{g b} .{ }^{1}$ With constraints in (5e), problem (5) is a mixed Boolean nonconvex program. Hence, the global optimal solution is not easy to achieve. It is worthy to mention that, even if the Boolean variables in (5) are fixed (or relaxed), the reduced problem is still NP-hard [1]. In addition, we recall that, to have solutions for problems of multicast systems, semidefinite relaxation (SDR) technique has been used to approximate the problems, e.g. [2], [4], [12]. However, this approach cannot be employed for (5) since the resulting problem is still nonconvex.

\section{Proposed SOLUtion}

In this section, to solve (5), we introduce an iterative procedure based on the state-of-the-art technique namely successive convex approximation (SCA) [13], [14], which has been widely used in wireless communication designs, e.g. [15], [16]. For this purpose, problem (5) needs to be transformed into a more amenable formulation. To do so, we first use DC function to equivalently represent the Boolean constraints in (5e) as [17, Chap. 4]

$$
d_{g b} \in\{0,1\} \Leftrightarrow\left\{\begin{array}{l}
d_{g b}-d_{g b}^{2} \leq 0 \\
0 \leq d_{g b} \leq 1
\end{array}\right.
$$

${ }^{1}$ The constraint shown in $(5 b)$ is for the case of total backhaul capacity. We remark that the method proposed in this paper can be straightforwardly applied to the case of individual transmitter backhaul links with constraint (5b) is rewritten as $\sum_{g=1}^{G} d_{g b} R_{g} \leq \bar{C}_{b}, \forall b$, where $\bar{C}_{b}$ denotes the backhaul capacity corresponding to transmitter $b$. 
In addition, let us introduce variable $\vartheta_{g}$ such that data rate transmitted to group $g$ is $\log \left(1+\vartheta_{g}\right)$. Then we can rewrite problem (5) into a more tractable form as

$$
\begin{aligned}
& \underset{\mathbf{w}_{g}, p_{g b}, d_{g b}, \vartheta_{g}, \tilde{\vartheta}}{\operatorname{maximize}} \tilde{\vartheta} \\
& \text { subject to } \frac{\left|\mathbf{h}_{g_{i}} \mathbf{w}_{g}\right|^{2}}{\sum_{k=1, k \neq g}^{G}\left|\mathbf{h}_{g_{i}} \mathbf{w}_{k}\right|^{2}+\sigma_{g_{i}}^{2}} \geq \vartheta_{g}, \forall g_{i} \\
& \vartheta_{g} \geq \tilde{\vartheta}, \forall g \\
& \sum_{g=1}^{G}\left(\sum_{b=1}^{B} d_{g b}\right) \log \left(1+\vartheta_{g}\right) \leq \bar{C} \\
&\left\|\mathbf{T}_{b} \mathbf{w}_{g}\right\|_{2}^{2} \leq d_{g b} p_{g b}, \forall g, b \\
& \sum_{g=1}^{G} p_{g b} \leq \bar{P}_{b}, \forall b \\
& d_{g b}-d_{g b}^{2} \leq 0, \forall g, b \\
& 0 \leq d_{g b} \leq 1, \forall g, b
\end{aligned}
$$

where all variables in (7) are continuous due to (6). However, problem (7) is still difficult to solve due to the intractable format of (7b), (7d). Thus we introduce slack variables $t_{g_{i}}>0$ and $s_{g} \geq 0$, then rewrite (7) as

$$
\begin{aligned}
& \text { maximize } \tilde{\vartheta} \\
& \vartheta_{g}, \vartheta, t_{g i}, s_{g} \\
& \text { subject to } \frac{\left|\mathbf{h}_{g_{i}} \mathbf{w}_{g}\right|^{2}}{t_{g_{i}}} \geq \vartheta_{g}, \forall g_{i} \\
& \sum_{k=1, k \neq g}^{G}\left|\mathbf{h}_{g_{i}} \mathbf{w}_{k}\right|^{2}+\sigma_{g_{i}}^{2} \leq t_{g_{i}}, \forall g_{i} \\
& \log \left(1+\vartheta_{g}\right) \leq s_{g}, \forall g \\
& \sum_{g=1}^{G}\left(\sum_{b=1}^{B} d_{g b}\right) s_{g} \leq \bar{C} \\
& \text { (7c), (7e), (7f), (7g), (7h) }
\end{aligned}
$$

At this stage, if we directly apply SCA to (8), there is some iteration where the subproblem is infeasible due to $(7 \mathrm{~g})$ and (7h) leading to the failure of obtaining solutions for (5). Inspired by the recent results in DC program [18], we tackle this issue by alternatively considering the relaxation of (8) given as

$$
\begin{aligned}
& \underset{\substack{\mathbf{w}_{g}, p_{g b}, d_{g b}, \vartheta_{g}, \tilde{\vartheta}, t_{g_{i}}, s_{g}, \phi_{g b}}}{\operatorname{maximize}} \tilde{\vartheta} \sum_{g, b} \phi_{g b} \\
& \text { subject to (7c), (7e), (7f), (7h), (8b), (8c), (8d), (8e) } \\
& \qquad d_{g b}-d_{g b}^{2} \leq \phi_{g b}, \forall g, b
\end{aligned}
$$

where $\phi_{g b} \geq 0, \forall g, b$, are slack variables and $\lambda>0$ is the penalty parameter. Obviously, (8) and (9) are equivalent when $\phi_{g b}=0, \forall g, b$. From now on, we introduce an iterative procedure based on SCA to solve (9) such that, at the convergence points, the Karush-Kuhn-Tucker (KKT) conditions of (9) are satisfied and $\phi_{g b}=0, \forall g, b$ (i.e. the convergence points are also the feasible solutions of (8)).

Let us first consider nonconvex constraint (8b). An important observation is that the left side of this constraint is a quadratic-over-linear function which is convex (c.f. [19, chap. 3]). For ease of description, let us define $u_{g_{i}} \triangleq \mathbf{h}_{g_{i}} \mathbf{w}_{g}$ and $u_{g_{i}}^{(n)} \triangleq \mathbf{h}_{g_{i}} \mathbf{w}_{g}^{(n)}$ where $\left(\mathbf{w}_{g}^{(n)}, p_{g b}^{(n)}, d_{g b}^{(n)}, \vartheta_{g}^{(n)}, \tilde{\vartheta}^{(n)}, t_{g_{i}}^{(n)}, s_{g}^{(n)}, \phi_{g b}^{(n)}\right)$ is supposed to be some feasible point of (9). Then an approximation of this constraint is given as [16]

$$
\begin{gathered}
\frac{2 \Re\left(\left(u_{g_{i}}^{(n)}\right)^{H}\left(u_{g_{i}}-u_{g_{i}}^{(n)}\right)\right)}{t_{g_{i}}^{(n)}}+\frac{\left(u_{g_{i}}^{(n)}\right)^{H} u_{g_{i}}^{(n)}}{t_{g_{i}}^{(n)}} \\
-\frac{\left(u_{g_{i}}^{(n)}\right)^{H} u_{g_{i}}^{(n)}}{\left(t_{g_{i}}^{(n)}\right)^{2}}\left(t_{g_{i}}-t_{g_{i}}^{(n)}\right) \geq \vartheta_{g}
\end{gathered}
$$

which is convex since the left side is linear in the involved variables. We now turn our attention on nonconvex constraint (8d). In the similar manner, since the left side is a logarithm function, we arrive at a convex approximation of this constraint given as

$$
\log \left(1+\vartheta_{g}^{(n)}\right)+\frac{\left(\vartheta_{g}-\vartheta_{g}^{(n)}\right)}{1+\vartheta_{g}^{(n)}} \leq s_{g}
$$

To deal with nonconvex constraint (8e), we introduce variables $v_{g}$, then equivalently rewrite the constraint as

$$
\left\{\begin{array}{l}
v_{g}=\sum_{b=1}^{B} d_{g b} \\
\sum_{g=1}^{G}\left(\left(v_{g}+s_{g}\right)^{2}-\left(v_{g}-s_{g}\right)^{2}\right) \leq 4 \bar{C}
\end{array}\right.
$$

where the first constraint is linear and the second one is nonconvex with the left side is the summation of difference of quadratic functions. We can convexly approximate the second constraint in (12) as follows

$$
\begin{aligned}
& \sum_{g=1}^{G}\left[\left(v_{g}+s_{g}\right)^{2}-\left(v_{g}^{(n)}-s_{g}^{(n)}\right)^{2}\right. \\
& \left.\quad-2\left(v_{g}^{(n)}-v_{g}^{(n)}\right)\left(v_{g}-v_{g}^{(n)}-s_{g}+s_{g}^{(n)}\right)\right] \leq 4 \bar{C}
\end{aligned}
$$

Finally, for the last nonconvex constraint (9c), we have a convex approximation given as

$$
\left(d_{g b}^{(n)}\right)^{2}+2 d_{g b}^{(n)}\left(d_{g b}-d_{g b}^{(n)}\right)+\phi_{g b} \geq d_{g b}
$$

We now present the proposed iterative procedure whose main steps are outlined in Algorithm 1. In particular, based on the above discussions, the safely approximated convex problem of (9) which is solved in the $(n+1)$ th iteration of 


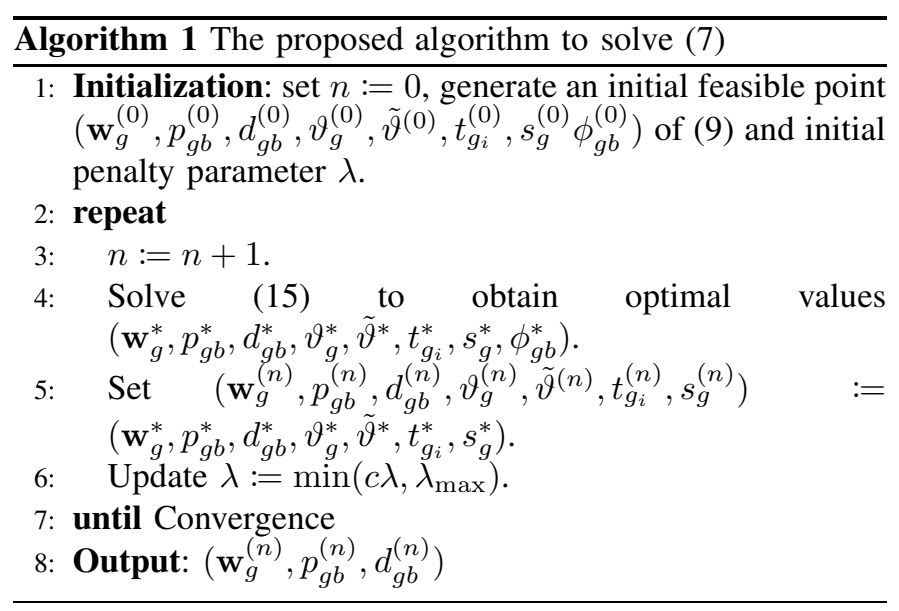

the algorithm (i.e. line 4) is given as

$$
\begin{aligned}
& \underset{\substack{\mathbf{w}_{g}, p_{g b}, d_{g b}, \vartheta_{g}, \tilde{\vartheta}, t_{g_{i}}, s_{g}, \phi_{g b}, v_{g}}}{\operatorname{maximize}} \tilde{\vartheta} \sum_{g, b} \phi_{g b} \\
& \quad \sqrt{\sum_{k=1, k \neq g}^{G}\left|\mathbf{h}_{g_{i}} \mathbf{w}_{k}\right|^{2}+\sigma_{g_{i}}^{2}+\frac{\left(t_{g_{i}}-1\right)^{2}}{4}} \leq \frac{t_{g_{i}}+1}{2}, \forall g_{i} \\
& \quad\left\|\left[\begin{array}{c}
\mathbf{T}_{b} \mathbf{w}_{g} \\
\frac{1}{2}\left(d_{g b}-p_{g b}\right)
\end{array}\right]\right\|_{2} \leq \frac{1}{2}\left(d_{g b}+p_{g b}\right), \forall g, b \\
& v_{g}=\sum_{b=1}^{B} d_{g b}
\end{aligned}
$$

where (15c) and (15d) are second-order cone representations of $(8 c)$ and (7e), respectively [20]. At the initial stage of the algorithm, the penalty parameter $\lambda$ is set at small value to provide high relaxation for the BS selection. Then, this parameter is increased after each iteration by a constant $c>1$ (line 6) to guarantee that $\sum_{g, b}\left|\phi_{g b}^{*}\right|=0$ when $n \rightarrow \infty$. Particularly, the property of the proposed algorithm (Algorithm 1 ) is stated in the following theorem.

Theorem 1. There exist finite values of $\lambda_{\max }$ and $n_{1}$ such that $\sum_{g, b}\left|\phi_{g b}^{*}\right|=0$ in iteration $n_{2}, \forall n_{2} \geq n_{1}$, and $\lim _{n \rightarrow \infty} \| \tilde{\vartheta}^{(n+1)}-$ $\tilde{\vartheta}^{(n)} \|_{2}=0$. In addition, the convergence points of Algorithm 1 satisfy the Karush-Kuhn-Tucker (KKT) conditions of (9).

Proof: Please refer to Appendix.

According to Theorem 1, Algorithm 1 is guaranteed to converge to stationary points of (8) (due to $\sum_{g, b}\left|\phi_{g b}^{*}\right|=0$ ). In general, $\lambda_{\max }$ is difficult to know. Thus, in practical implementations, we can increase $\lambda$ until $\sum_{g, b}\left|\phi_{g b}^{*}\right|<\epsilon$ is satisfied, then terminate the procedure when $\left\|\tilde{\vartheta}^{(n+1)}-\tilde{\vartheta}^{(n)}\right\|_{2}<\epsilon$. Herein, $\epsilon \approx 0$ is positive tolerance parameter.

Complexity analysis: We now discuss the computational complexity of Algorithm 1. Since there is no theoretical result about the number of required iterations for the convergence of the SCA-based approaches, we focus on the arithmetical cost in an iteration which is dominated by that of solving (15). We note that problem (15) is a SOCP which requires less computational effort to be solved compared to other nonlinear programs (e.g. semidefinite and generic convex programs) [20]. To be specific, the number of required iterations satisfying a given acceptable tolerance (when solving (15) by an interior-point method) is bounded by $\mathcal{O}(\sqrt{G B+U})$ and the bound of arithmetical cost in one iteration (of the interior-point method) is $\mathcal{O}\left(G^{3}\left(B+\sum_{b} L_{b}\right)^{2}\left(U+B+\sum_{b} L_{b}\right)\right)$.

\section{NumericAl RESUlts}

In this section, we report simulation results to evaluate the performances of the proposed method. For comparison purposes, we additionally provide the performances of two other strategies. The first strategy is the coordinated beamforming $(\mathrm{CoB})$ where each group receives data from only one transmitter [4]. The second one is the joint beamforming (JB) where each group is served by all transmitters. The solution for JB is obtained based on that in [2], [12]. To solve the convex problems in this section, we use the modeling package YALMIP [21] with the inner solver SeDuMi [22].

We consider the simulation model as follows. For each group, the positions of receivers are randomly generated in a circle with a radius of 10 meters. In a similar manner, the positions of transmitters and that of the centers of multicast groups are randomly generated inside a circle with a radius of 200 meters. The path loss model follows the one in 3GPP, i.e. $\operatorname{PL}(\mathrm{dB})=30.18+26 \log _{10}(l)$ where $l$ is the distance in meters [23]. The log normal shadowing standard deviation is 4 , the noise power density is $-150 \mathrm{dBm} / \mathrm{Hz}$, and the system bandwidth is $1 \mathrm{MHz}$. Other specific parameters are written in the captions of figures.

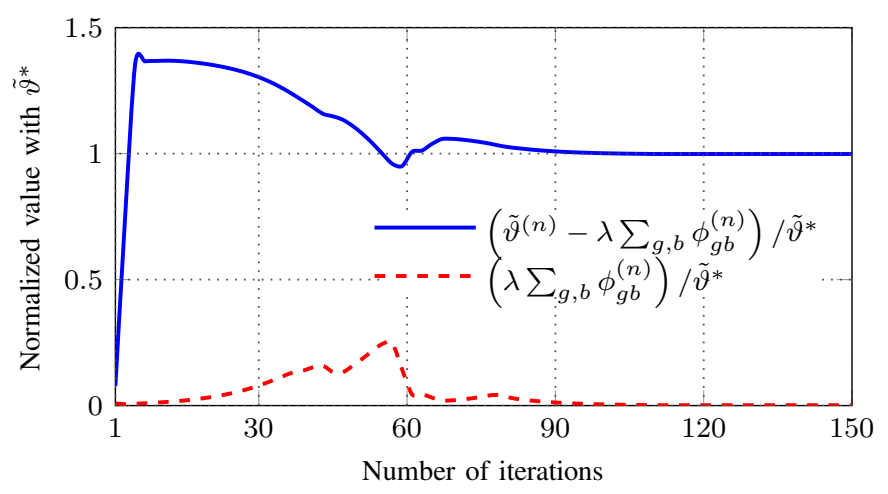

Figure 2. Convergence performance of Algorithm 1 over a random channelization. The network setting is $G=3, B=4, L_{b}=4, \bar{C}=$ 15 (Mnats/s), $\bar{P}_{b}=2 \mathrm{dBm} \forall b$, and the number of users in each group is 3 (i.e. $U=9$ ).

In the first experiment, we numerically study the convergence of the proposed algorithm. Fig. 2 plots the values of functions $\left(\tilde{\vartheta}^{(n)}-\lambda \sum_{g, b} \phi_{g b}^{(n)}\right) / \tilde{\vartheta}^{*}$ and $\left(\lambda \sum_{g, b} \phi_{g b}^{(n)}\right) / \tilde{\vartheta}^{*}$, where $\tilde{\vartheta}^{*}$ is the obtained value of $\tilde{\vartheta}$ at a convergence point, over the running iterations. As we can observe in the figure, the algorithm converges in this case and the objective function of (15) arrives at $\tilde{\vartheta}^{*}$ when convergence due to $\lambda \sum_{g, b} \phi_{g b}^{(n)} \approx 0$. 


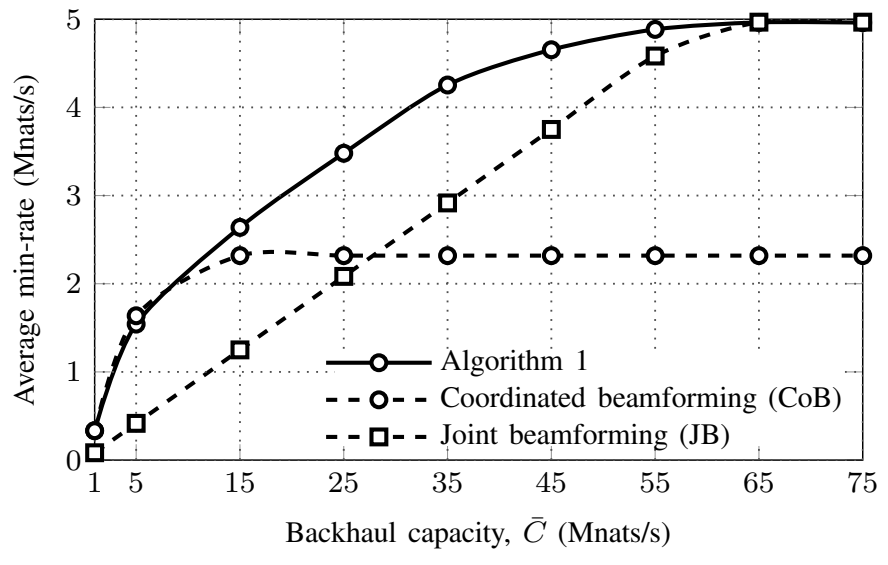

Figure 3. Average min-rate versus the backhaul capacity $\bar{C}$. The network setting is $G=3, B=4, L_{b}=4, \bar{P}_{b}=10 \mathrm{dBm} \forall b$, and the number of users in each group is 3 .

In Fig. 3, we investigate the achievable min-rate performances of the considered schemes, i.e. Algorithm 1, CoB and $\mathrm{JB}$, versus the backhaul capacity $\bar{C}$. The results in the figure clearly show that the min-rate of all considered schemes increases (to the limitations corresponding to unlimited backhaul models) when $\bar{C}$ increases. We can also see in the figure that CoB scheme achieves better min-rate than the JB scheme when $\bar{C}$ is small and vice versa when $\bar{C}$ is large. In other words, JB outperforms $\mathrm{CoB}$ when the backhaul capacity is large enough. We can explain this observation as follows. When $\bar{C}$ is small, the achievable min-rate of the wireless transmissions is larger than the limitation of the backhaul link, i.e. the min-rate of the network strongly depends on the transmitted data rate from central processor to transmitters (via backhaul). On the other hand, as $\bar{C}$ is large, transmission schemes and transmit power budgets have the main impact on the network capacity. This also explains the fact that the performance of the proposed method is close to that of $\mathrm{CoB}$ and JB as $\bar{C}$ is small and large, respectively. As expected, the proposed method yields good performances in all cases of $\bar{C}$.

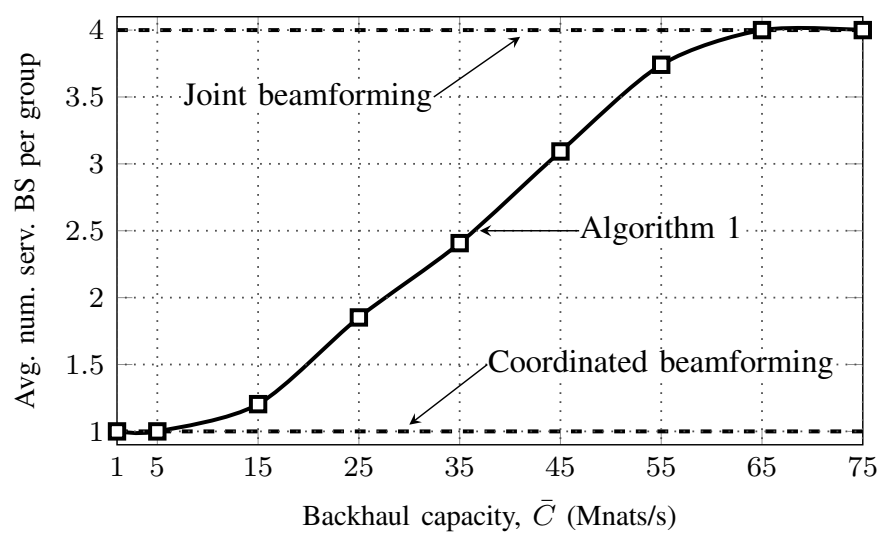

Figure 4. Average number of serving BSs per group versus the backhaul capacity $\bar{C}$. The setting network is same as that in Fig. 3 .

Fig. 4 depicts the average number of serving transmitters per multicast group versus the backhaul capacity $\bar{C}$. As can be observed, the results shown in this figure are entirely consistent with those presented in Fig. 3. The min-rate of the network in low backhaul capacity regime relies on data rate transmitted over backhaul link, thus, the strategy is to use a small number of transmitters to transfer data to a group. On the contrary, at the high backhaul capacity regime, the min-rate is limited by wireless transmissions. Therefore, to improve the min-rate, the proposed method should use more transmitters (per group).

\section{CONClusion}

We have investigated the cooperative multicell networks where multiple BSs collaborate to simultaneously transmit data to multiple multicast groups under the limitation of the backhaul link capacity. The target of this paper is to design the low-complexity scheme of transmitter selection and linear precoding such that the minimum data rate among the groups is maximized. The problem is cast as a mixed Boolean nonconvex program, and thus, we have transformed it into the equivalent, but more tractable, program using DC functions. Then, we have proposed an iterative algorithm which provably converges to stationary points of this problem. Simulation results have confirmed the effectiveness of our proposed method.

\section{APPENDIX}

Let $\varphi_{g b}^{(n)}$ and $\rho_{g b}^{(n)}$ be the KKT multipliers corresponding to the constraints (14) and $\phi_{g b} \geq 0$, respectively, in iteration $n$. Since the Slater's condition is satisfied in (15), we have

$$
-\lambda+\varphi_{g b}^{(n+1)}+\rho_{g b}^{(n+1)}=0
$$

In addition, following the same arguments in [24], we have $\sum_{g, b}\left|\varphi_{g b}^{(n)}\right|$ is upper bounded. Therefore, if $\lambda_{\max }$ is larger than this bound, there exists $n_{1}$ such that $-\lambda+\varphi_{g b}^{(n+1)}<0, \forall n>$ $n_{1}$. Combining with (16), we have $\rho_{g b}^{(n+1)}>0, \forall n>n_{1}$. Moreover, following the KKT conditions, we have $\rho_{g b}^{(n)} \phi_{g b}^{(n)}=$ $0, \forall n$. Therefore, finally, we have $\phi_{g b}^{(n+1)}=0, \forall n>n_{1}$.

Now, for arbitrary $n_{2}$ such that $n_{2}>n_{1}$, we have $\tilde{\vartheta}^{\left(n_{2}+1\right)} \geq$ $\tilde{\vartheta}^{\left(n_{2}\right)}$ due to the fact that the solution in iteration $n_{2}$ is also a feasible point in iteration $\left(n_{2}+1\right)$. We note that $\tilde{\vartheta}$ is upper bounded due to the power and backhaul constraints. Therefore, we have the conclusion that $\lim _{n \rightarrow \infty}\left\|\tilde{\vartheta}^{(n+1)}-\tilde{\vartheta}^{(n)}\right\|_{2}=0$. In addition, following the same arguments in [13], we have the fact that the convergence points of Algorithm 1 satisfy the KKT conditions of (9). This completes the proof.

\section{ACKNOWLEDGMENT}

This work was supported in part by the Academy of Finland under project Message and CSI Sharing for Cellular Interference Management with Backhaul Constraints (MESIC) belonging to the WiFIUS program with NSF. 


\section{REFERENCES}

[1] N. Sidiropoulos, T. Davidson, and Z.-Q. Luo, "Transmit beamforming for physical-layer multicasting," IEEE Trans. Signal Process., vol. 54, no. 6, pp. 2239-2251, June 2006.

[2] D. Christopoulos, S. Chatzinotas, and B. Ottersten, "Weighted fair multicast multigroup beamforming under per-antenna power constraints," IEEE Trans. Signal Process., vol. 62, no. 19, pp. 5132-5142, Oct. 2014.

[3] D. Lecompte and F. Gabin, "Evolved multimedia broadcast/multicast service (eMBMS) in LTE-advanced: overview and rel-11 enhancements," IEEE Commun. Mag., vol. 50, no. 11, pp. 68-74, Nov. 2012.

[4] Z. Xiang, M. Tao, and X. Wang, "Coordinated multicast beamforming in multicell networks," IEEE Trans. Wireless Commun., vol. 12, no. 1, pp. 12-21, January 2013.

[5] A. H. Phan, H. D. Tuan, H. H. Kha, and D. T. Ngo, "Nonsmooth optimization for efficient beamforming in cognitive radio multicast transmission," IEEE Trans. Signal Process., vol. 60, no. 6, pp. 29412951, June 2012.

[6] D. Gesbert, S. Hanly, H. Huang, S. Shamai Shitz, O. Simeone, and W. Yu, "Multi-cell MIMO cooperative networks: A new look at interference," vol. 28, no. 9, pp. 1380-1408, Dec. 2010.

[7] O. Tipmongkolsilp, S. Zaghloul, and A. Jukan, "The evolution of cellular backhaul technologies: Current issues and future trends," "IEEE Commu. Surveys Tutorials", vol. 13, no. 1, pp. 97-113, 2011.

[8] B. Dai and W. Yu, "Sparse beamforming and user-centric clustering for downlink cloud radio access network,' IEEE Access, vol. 2, pp. 13261339, 2014.

[9] F. Zhuang and V. Lau, "Backhaul limited asymmetric cooperation for MIMO cellular networks via semidefinite relaxation," IEEE Trans. Signal Process., vol. 62, no. 3, pp. 684-693, Feb 2014.

[10] H. Zhou, M. Tao, E. Chen, and W. Yu, "Content-centric multicast beamforming in cache-enabled cloud radio access networks," in Proc. IEEE Global Commu. Conf. (GLOBECOM), Dec. 2015.

[11] J. Zhao, T. Quek, and Z. Lei, "Coordinated multipoint transmission with limited backhaul data transfer," IEEE Trans. Wireless Commun., vol. 12, no. 6, pp. 2762-2775, June 2013

[12] E. Karipidis, N. Sidiropoulos, and Z.-Q. Luo, "Quality of service and max-min fair transmit beamforming to multiple cochannel multicast groups," IEEE Trans. Signal Process., vol. 56, no. 3, pp. 1268-1279, March 2008.

[13] A. Beck, A. Ben-Tal, and L. Tetruashvili, "A sequential parametric convex approximation method with applications to nonconvex truss topology design problems," Journal of Global Optimization, vol. 47, no. 1 , pp. 29-51, 2010.

[14] B. R. Marks and G. P. Wright, "A general inner approximation algorithm for nonconvex mathematical programs," Operations Research, vol. 26, no. 4, pp. 681-683, August 1978.

[15] L.-N. Tran, M. Hanif, and M. Juntti, "A conic quadratic programming approach to physical layer multicasting for large-scale antenna arrays," IEEE Signal Process. Lett., vol. 21, no. 1, pp. 114-117, Jan 2014.

[16] Q.-D. Vu, L.-N. Tran, R. Farrell, and E.-K. Hong, "An efficiency maximization design for SWIPT," IEEE Signal Process. Lett., vol. 22, no. 12, pp. 2189-2193, Dec 2015.

[17] H. Tuy, Convex Analysis and Global Optimization. Springer US, 1998.

[18] T. P. Dinh and H. A. L. Thi, "Recent advances in dc programming and DCA," Transactions on Computational Intelligence XIII, vol. 8342, pp. 1-37, April 2014.

[19] S. Boyd and L. Vandenberghe, Convex optimization, 1st ed., S. Boyd, Ed. Cambridge, 2004

[20] M. Lobo, L. Vandenberghe, S. Boyd, and H. Lebret, "Applications of second-order cone programming," Linear Algebra Appl., Special Issue on Linear Algebra in Control, Signals and Image Processing, pp. 193228, Nov. 1998.

[21] J. Löfberg, "YALMIP : A toolbox for modeling and optimization in MATLAB," in Proc. the CACSD Conference, Taipei, Taiwan., 2004. [Online]. Available: http://users.isy.liu.se/johanl/yalmip

[22] J. F. Sturm, "Using SeDuMi 1.02, a MATLAB toolbox for optimization over symmetric cones," Optim. Meth. Softw., vol. 11, pp. 625-653, 1999.

[23] 3GPP, "Spatial channel model for multiple input multiple output (MIMO) simulations," 3rd Generation Partnership Project, TR 25.996. [Online]. Available: http://www.3gpp.org/technologies

[24] T. V. Do, H. A. L. Thi, and N. T. Nguyen, Advanced Computational Methods for Knowledge Engineering. Springer, 2014. 